\title{
BCG lymphadenopathy detected in a BCG-vaccinated infant
}

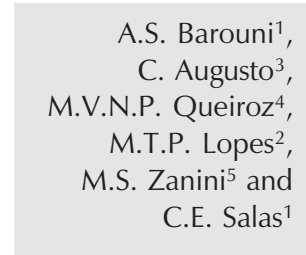

Correspondence

C.E. Salas

Laboratório de Biologia Molecular

de Produtos Naturais

ICB, UFMG

Av. Antonio Carlos, 6627

31270-010 Belo Horizonte, MG

Brasil

Fax: +55-31-3499-2646

E-mail: cesbufmg@mono.icb.ufmg.br

Research supported by CNPq, FAPEMIG and the Lybia Secretary of Education.

Received September 22, 2003 Accepted December 4, 2003 ......................

\author{
Departamentos de ${ }^{1}$ Bioquímica e Imunologia and ${ }^{2}$ Farmacologia, \\ Instituto de Ciências Biológicas, Universidade Federal de Minas Gerais, \\ Belo Horizonte, MG, Brasil \\ ${ }^{3}$ Fundação Ezequel Diaz and ${ }^{4}$ Departamento de Pediatria, \\ Faculdade de Ciências Médicas, Belo Horizonte, MG, Brasil \\ ${ }^{5}$ Centro de Ciências Agrárias, Universidade Federal do Espírito Santo, \\ Alegre, ES, Brasil
}

\begin{abstract}
Large-scale vaccination with BCG, the live attenuated strain of Mycobacterium bovis, is being adopted around the world, although sporadic complications have occurred after the procedure. Lymphadenopathy is not uncommon especially in babies under one year $(0.73 \%$ of vaccinated infants), but the swelling subsides within 2 months in most cases, with no medical or surgical treatment. Brazil adopted BCG vaccination program earlier in the seventies and by 1995 more than $96 \%$ of the infant population received this immunization. We report here the occurrence of lymphadenopathy in a two-year-old child vaccinated with the Brazilian BCG strain. The diagnosis was made using a lymph node biopsy and intestinal aspirates that yielded a positive mycobacterial culture. The isolate was resistant to isoniazid, rifampicin, pyrazinamide and thiophen-2-carbonic acid hydrazide, sensitive to streptomycin, ethambutol, and p-nitrobenzoic acid, and reacted positively to cyclo-serine and negatively to niacin. The pncA gene involved in bacterial activation of pyrazinamide contains in $M$. bovis a point mutation that renders pyrazinamidase unable to catalyze drug activation. Therefore, this polymorphism is a good option for developing methods to differentiate $M$. bovis and $M$. tuberculosis. Taking advantage of this difference we further analyzed the isolates by single-stranded conformation polymorphism electrophoresis of DNA following PCR of the pncA gene. The isolate identity was confirmed by RFLP electrophoretic analysis of the amplified fragment following Eco065I digestion, which selectively cleaves $M$. tuberculosis DNA. From this result it is proposed that RFLP of $p n c A$ gene represents an alternative for differential diagnosis of $M$. bovis.
\end{abstract}

The Bacille Calmette-Guérin (BCG) vaccine has been administered percutaneously in Brazil since 1968 using the multiple puncture method. In 1995 approximately $96 \%$ of all infants aged 12 to 23 months received
Key words

- Bacille Calmette-Guérin (BCG)

- Vaccination

- Polymerase chain reaction

- Lymphadenopathy

- Antibiotic resistance immunization against BCG. The side effects of the BCG vaccination program include lymphadenopathy in very young infants or osteomyelitis resulting from generalized dissemination of BCG (1-3). A prior study re- 
ports that the frequency of lymphadenopathy among vaccinated infants is $0.73 \%$, especially in babies under one year (4). BCG osteomyelitis has also been reported as a complication of intravesical BCG therapy for bladder carcinoma (1). The definitive diagnosis of each case is made by the recovery of the BCG strain of Mycobacterium bovis from the affected focus. However, the identification process is not simple because it relies on the isolation of the bacteria.

A 2-year-old Brazilian boy who had received BCG vaccination at the age of 1 month had a history of congenital neural disorders and of early pustular axillary nodes detected at the age of 3 months. At the age of 5 months, he developed a pustular cervical abscess and was treated with clindamycin. At the age of 15 months he was admitted to the Santa Casa Hospital of Belo Horizonte. After a lymph node biopsy was obtained, the child was submitted to treatment with oxacillin and amikacin over a period of $48 \mathrm{~h}$.

Ganglionic tuberculosis was suspected based on acid-fast bacilli detected after ZiehlNeelsen staining of the biopsy. The patient was treated with a cocktail of rifampicin, isoniazid and pyrazinamide for 37 days without improvement of clinical symptoms.

Two intestinal washes and lymph node aspirates revealed the presence of acid-resistant bacteria. Histologic section analysis suggested the presence of atypical tuberculosis without a granulocytic reaction.

Treatment with ethambutol plus ciprofloxacin and clarithromycin induced lymph node regression and healing of an axillary fistula. However, the fistular symptoms reappeared before completion of treatment.

There has been concern about the administration of live BCG to persons infected with $\mathrm{HIV}$, although HIV-related complications arising from BCG vaccination are limited. Most cases involve infants previously infected with HIV and occur within short intervals between BCG vaccination and the onset of symptoms (5). To rule out this possibility, serum samples from the present patient were tested for HIV, yielding a negative result.

Mycobacterium was identified in the lymph node biopsy by culture in Lowenstein Jensen medium. Antibiotic resistance of the isolated bacteria was assessed by the multiple proportion procedure with colony scoring 28 days after seeding. The isolate was resistant to isoniazid, rifampicin, pyrazinamide and thiophen-2-carbonic acid hydrazide and sensitive to streptomycin, ethambutol, and p-nitrobenzoic acid, with a positive reaction to cyclo-serine and a negative reaction to niacin.

Interestingly, a loss of virulence of $M$. bovis in guinea pigs and cattle is linked to isoniazid resistance $(6,7)$, while the appearance of multidrug resistance in immunodeficient humans infected with $M$. bovis is unusually high. This seems to be the case in the present report, in which the isolate was found to be multidrug isoniazid resistant. This selection of this resistance may have been induced by prior treatment with isoniazid. The increasing number of isoniazid-resistant tubercles in both HIV-positive and -negative patients suggests that at least some of these resistant organisms retain or enhance their virulence after gene mutation.

DNA was obtained from the isolates after culture in Lowenstein Jensen medium for 28 days at $37^{\circ} \mathrm{C}$. DNA samples were extracted in the presence of proteinase $\mathrm{K}$ (8). Mycobacterium complex was identified by PCR of the $p n c A$ gene using primers $\mathrm{P} 3$ and $\mathrm{P} 4$ (9). DNA (5-50 ng) was amplified following denaturation in buffer containing $50 \mathrm{mM} \mathrm{KCl}$, $1.5 \mathrm{mM} \mathrm{MgCl}_{2}, 10 \mathrm{mM}$ Tris-Cl, $\mathrm{pH} 8$, and $0.01 \%$ gelatin at $95^{\circ} \mathrm{C}$ for 30 cycles, by annealing at $68^{\circ} \mathrm{C}$ for $1 \mathrm{~min}$ and extension at $72^{\circ} \mathrm{C}$ for 1 min with $2.5 \mathrm{U}$ Taq polymerase in the presence of primers P3 (5'ATCAGCG ACTACCTGGCCGA $3^{\prime}$ ) and P4 (5'GATTG CCGACGTGTCCAGAC 3'). Approximately, $1 / 4$ of the PCR product was submitted to $6 \%$ PAGE under non-denaturing conditions to confirm the presence of the 180-bp 
fragment. As expected, a main DNA band of 180 bp characteristic of the Mycobacterium complex was identified by $6 \%$ PAGE (data not shown).

To further characterize the source of the DNA fragment, we used SSCP 20\% PAGE employing M. bovsis AN5 and H37Rv (ATCC) as standards. One half of the remaining $(10 \mu \mathrm{l})$ sample was denatured with $95 \%$ formamide, $20 \mathrm{mM}$ EDTA, $0.05 \%$ bromophenol blue, and $0.05 \%$ xylene cyanol at $100^{\circ} \mathrm{C}$ for $5 \mathrm{~min}$ and submitted to PAGE for the SSCP experiments. Electrophoresis was carried out at $300 \mathrm{~V}, 20$ watts for $12 \mathrm{~h}$ at $7^{\circ} \mathrm{C}$ in $0.5 \mathrm{X}$ TBE followed by staining with silver nitrate.

Due to the presence of a polymorphic site at position 169 of the $p n c A$ gene, it is possible to differentiate between BCG and H37Rv $M$. tuberculosis DNAs (Figure 1), i.e., the profile of the clinical sample is similar to that of BCG and different from that of M. tuberculosis. We further confirmed this difference by RFLP, since the allelic variant in $M$. bovis can be recognized and cleaved by the enzyme Eco065I when $\mathrm{C}_{169}$ is present (Barouni AS, Augusto CJ, Lopes MTP, Zanini MS and Salas CE, unpublished results). The result in Figure 2 confirms that the patient isolate corresponds to M. bovis. Although solid evidence is scanty, BCG lymphoadenopathy is mainly attributed to BCG vaccination, particularly in immunodeficient subjects. It is also possible that some batches of the vaccine contain incompletely attenuated bacteria, thus facilitating their acti- vation after immunization.

We conclude that this alternative differential diagnosis is reliable since a screening of more than 50 clinical samples from patients infected with M. bovis or M. tuberculosis matched the expected profile. This result supports the diagnostic use of the pncA gene to differentiate between $M$. bovis and M. tuberculosis.

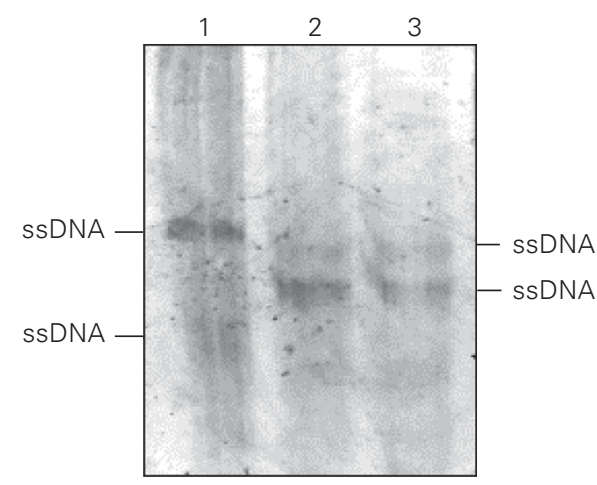

Figure 1. SSCP PAGE after PCR of the pncA gene within the polymorphic region. Lane 1 , Mycobacterium tuberculosis H37Rv(ATCC); lane 2, M. bovis (AN5); lane 3, sample from patient isolate. The sample was denatured for $5 \mathrm{~min}$ at $100^{\circ} \mathrm{C}$ and after cooling was applied to $20 \%$ polyacrylamide gels. The electrophoresis was run at 300 $\mathrm{V}, 25 \mathrm{~mA}$, for $14 \mathrm{~h}$ at $7^{\circ} \mathrm{C}$. Following electrophoresis the gel was developed by silver staining.

Figure 2. Electrophoresis of the pncA amplicon digested with Eco65OI. DNA samples were amplified with primers P3 and P4 and digested with Eco065I as described in Methods. Lanes $a, b, M$. tuberculosis clinical isolates; lane $c$, sample from patient isolate; lane $d, M$. bovis (AN5); lane e, M. tuberculosis H37Rv(ATCC); lane $m, 100-b p$ molecular mass standard. The electrophoresis was run at 100 $\mathrm{V}, 6 \mathrm{~mA}$, for $60 \mathrm{~min}$ without cooling. Following electrophoresis the gel was developed by silver staining.

\section{References}

1. Civen R, Berlin G \& Panosian C (1994). Vertebral osteomyelitis after intravesical administration of Bacille Calmette-Guerin. Clinical Infectious Diseases, 18: 1013-1014.

2. Nishi J, Kamenososno A, Sarker KP, Yoshino S, Ikei J \& Matsuda Y (1997). Bacille Calmette-Guerin osteomyelitis. Pediatric Infectious Disease Journal, 16: 332-333.

3. Su WJ, Huang CY, Huang CY \& Perng RP (2001). Utility of PCR assay for rapid diagnosis of BCG infection in children. International Journal of Tuberculosis and Lung Disease, 5: 380-384.

4. Mori T, Yamauchi Y \& Shiozawa K (1996) Lymph node swelling due to Bacille Calmette-Guerin vaccination with multipuncture method. Tuberculosis and Lung Disease, 77: 269-273.

5. van Deutekom H, Smulders YM, Roozendaal KJ \& van Soolinge D (1996). Bacille Calmette-Guerin meninigitis in an AIDS patient 12 years after vaccination with BCG. Clinical Infectious Diseases, 22: 870-871.

6. Li Z, Kelley C, Collins F, Rouse D \& Morris S (1998). Expression of katG in Mycobacterium tuberculosis is associated with its growth and persistence in mice and guinea pigs. Journal of Infectious Diseases, 177: 1030-1035. 
7. Wedlock DN, Aldwell FE, Collins DM, de Lisle GW, Wilson T \& Buddle BM (1999). Immune responses induced in cattle by virulent and attenuated Mycobacterium bovis strains: correlation of delayed-type hypersensitivity with ability of strains to grow in macrophages. Infection and Immunity, 67: 2172-2177.

8. Zanini MS, Moreira EC, Lopes MT, Mota P \& Salas CE (1998). Detection of Mycobacterium bovis in milk by polymerase chain reaction. Journal of Veterinary Medicine. Series B: Infectious Diseases and Veterinary Public Health, 45: 473-479.

9. Scorpio A, Collins D, Whipple D, Cave D, Bates J \& Zhang Y (1997). Rapid differentiation of bovine and human tubercle bacilli based on a characteristic mutation in the Bovis pyrazinamidase gene. Journal of Clinical Microbiology, 35: 106-110. 